Research Article

\title{
Semi-G-Preinvexity and Optimality in Mathematical Programming
}

\author{
Zai Yun Peng, ${ }^{1}$ Yong Zhao, ${ }^{2}$ Kai Zhi Yu, ${ }^{3}$ and Zhi Lin ${ }^{1}$ \\ ${ }^{1}$ College of Science, Chongqing JiaoTong University, Chongqing 400074, China \\ ${ }^{2}$ Department of Mathematics, Chongqing Normal University, Chongqing 400013, China \\ ${ }^{3}$ Statistics School, Southwestern University of Finance and Economics, Chengdu 611130, China
}

Correspondence should be addressed to Zai Yun Peng; pengzaiyun@126.com

Received 28 February 2014; Accepted 17 March 2014; Published 7 April 2014

Academic Editor: Xian-Jun Long

Copyright (C) 2014 Zai Yun Peng et al. This is an open access article distributed under the Creative Commons Attribution License, which permits unrestricted use, distribution, and reproduction in any medium, provided the original work is properly cited.

\begin{abstract}
We mainly discuss the properties and applications of semi-G-preinvexity, which was introduced in Peng and Chang 2013. Examples are given to show the existence of semi-G-preinvex functions. Three important properties and characterizations of semi- $G$-preinvex functions are established. Furthermore, under semi-G-preinvexity, some optimality results are obtained in nonlinear programming problems with inequality constraint. Our results improve and generalize some known results.
\end{abstract}

\section{Introduction}

It is well known that the research on convexity and generalized convexity is one of the most important aspects in mathematical programming and optimization theory; see, for example, [1-5]. In fact, there are a number of nonlinear programming problems whose objective and constraints functions are nonconvex. Therefore, in the recent years, attempts are made by several authors to define various nonconvex classes of functions and to study their optimality criteria in solving such types of problems; see, for example, [6-14]. One of such a generalization of convex function is invexity notion introduced in [13] and called in [15]. Over the years, many generalizations of this concept have been given in the literature. In 1972, Avriel [16] introduced the definition of $r$-convex functions, which is a generalization of convex functions, and discussed some characterizations of $r$-convex functions. Later, Ben-Israel and Mond [17] considered a class of nondifferentiable functions that were called preinvex in [18] as a generalization of convexity and studied how and where preinvexity can replace convexity in multiobjective programming problems. Then, the concept of preinvexity was generalized to $B$-preinvexity [19] and semipreinvexity [20]. Using the definition of a weighted $r$-mean (where $r$ is a real number) for a sequence of positive numbers,
Antczak [21] introduced new classes of functions and called them $(p, r)$-preinvex with respect to $\eta$. Moreover, Antczak [22] considered a class of $r$-preinvex functions, which is a generalization of $r$-convex functions, and obtained some optimality results under appropriate $r$-preinvexity conditions for constrained optimization problems. In 2011, Zhao et al. [23] introduced the definition of $r$-semipreinvex functions and obtained some basic characterizations and optimality results in nonlinear programming.

On the other hand, Avriel et al. [24] introduced the definition of $G$-convex functions, which is another generalization of convex functions, and obtained some characterizations and the relations between $G$-convexity and convexity, where $G$ is a continuous real-valued increasing function. As a generalization of $G$-convexity and invexity, Antczak [25] introduced the concept of $G$-invexity and obtained some optimality conditions for constrained optimization problems under assumptions of $G$-invexity. Recently, Antczak [26] introduced the definition of $G$-preinvex functions, which is a generalization of $r$-preinvex functions with $r>0$ [22] with respect to the same $\eta$. Then, by using the G-preinvexity, some optimality results for constrained optimization problems were derived. Very recently, Peng and Chang [27] introduced a class of nonconvex functions, semi- $G$-preinvex functions, which is a generalization of $G$-preinvex functions 
[26], semipreinvex functions [20, 28], and $r$-semipreinvex functions with $r>0$ [23] with respect to the same $\eta$, and then they obtained some interesting properties of semi-Gpreinvex functions.

But, to the best of our knowledge, there are few optimality results in nonlinear programming (especially, in mathematical programming with constant) that have been concerned under semi-G-preinvex functions in the literature. So, in this paper, we mainly discuss the characterizations and applications in nonlinear programming of semi-G-preinvexity.

The rest of the paper is organized as follows. In Section 2, we recall some concepts and give some examples to show the existence of semi-G-preinvex functions. In Section 3, we establish three important properties and characterizations of semi-G-preinvex functions, which include that the ratio of semi-G-preinvex functions is semi-preinvex function. In Section 4, under semi-G-preinvexity, we obtain some optimality results in nonlinear programming problems with inequality constraint. Some examples are given for illustration of our results. Our results extend and generalize the corresponding ones in the literature [20, 26-28].

\section{Preliminaries}

Throughout this paper, let $X$ be a nonempty subset of $R^{n}$. Let $f: X \rightarrow R$ be a real-valued function and $\eta: X \times X \rightarrow R^{n}$ a vector-valued function. And let $I_{f}(X)$ be the range of $f$, that is, the image of $X$ under $f$, and let $f^{-1}$ be the inverse of $f$.

Now we recall some useful definitions.

Definition 1 (see [24]). Let $G$ be a continuous real-valued strictly monotonic function defined on $D \subset R$, and $f: X \rightarrow$ $R$, where $X$ is a nonempty convex set in $R^{n} . f$ is said to be $G$-convex on $X$ if and only if, for any $x, y \in X, \lambda \in[0,1]$,

$$
f(y+\lambda(x-y)) \leq G^{-1}(\lambda G(f(x))+(1-\lambda) G(f(y))),
$$

where $G^{-1}$ is the inverse of $G, f(X) \subset D$.

Remark 2. All convex functions are $G$-convex functions with $G(x)=x$, but the converse is not necessarily true.

Weir et al. $[5,18]$ presented the concepts of invex sets and preinvex functions which are generalization of convex sets and convex functions, respectively, as follows.

Definition 3. A set $X$ is said to be invex if there exists a vectorvalued function $\eta: X \times X \rightarrow R^{n}$ such that

$$
x, y \in X, \quad \lambda \in[0,1] \Longrightarrow y+\lambda \eta(x, y) \in X
$$

Definition 4. Let $K \subseteq R^{n}$ be an invex set with respect to $\eta$ : $K \times K \rightarrow R^{n}$ and let $f: K \rightarrow R$ be a mapping. We say that $f$ is preinvex if and only if

$$
\begin{array}{rr}
f(y+\lambda \eta(x, y)) \leq \lambda f(x)+(1-\lambda) f(y), \quad & \forall x, y \in K, \\
& \lambda \in[0,1] .
\end{array}
$$

Antczak presented the concepts of $G$-invex functions [25] and $G$-preinvex functions [26] which are generalization of $G$-convex functions and preinvex functions, respectively, as follows.

Definition 5. Let $X$ be a nonempty invex (with respect to $\eta$ ) subset of $R^{n}$ and let $f: X \rightarrow R$ be a differentiable function defined on $X$. Further, we assume that there exists a differentiable real-valued increasing function $G: I_{f}(X) \rightarrow$ $R$. Then $f$ is said to be $G$-invex at $y$ on $X$ with respect to $\eta$ if there exists a vector-valued function $\eta: X \times X \rightarrow R^{n}$ such that, for all $x \in X$,

$$
G(f(x))-G(f(y)) \geq G^{\prime}(f(y)) \nabla f(y) \eta(x, y) .
$$

Definition 6. Let $X$ be a nonempty invex (with respect to $\eta$ ) subset of $R^{n} ; f: X \rightarrow R$ is said to be (strictly) $G$-preinvex at $y$ with respect to $\eta$ if and only if there exist a continuous real-valued increasing function $G: I_{f}(X) \rightarrow R$ and a vector-valued function $\eta: X \times X \rightarrow R^{n}$ such that, for all $x \in X(x \neq y)$ and any $\lambda \in[0,1](\lambda \in(0,1))$,

$$
\begin{aligned}
f(y & +\lambda \eta(x, y)) \\
& \leq G^{-1}(\lambda G(f(x))+(1-\lambda) G(f(y)))(<) .
\end{aligned}
$$

If the inequality above is satisfied for any $y \in X$, then $f$ is (strictly) $G$-preinvex on $X$ with respect to $\eta$.

Remark 7. Every $r$-preinvex function with respect to $\eta$ with $r>0$ introduced in [22] is G-preinvex with respect to the same function $\eta$, where $G: I_{f}(X) \rightarrow R$ is defined by $G(t)=$ $e^{r t}$. Every preinvex function with respect to $\eta$ is $G$-preinvex function with respect to the same $\eta$, where $G(x)=x$.

Definition 8 (see [20]). $X \subseteq R^{n}$ is said to be semiconnected set if there exists $\eta: X \times X \times[0,1] \rightarrow R^{n}$ such that, for any $x, y \in X, \lambda \in[0,1], y+\lambda \eta(x, y, \lambda) \in X$.

Definition 9 (see $[20,28]$ ). Let $X \subseteq R^{n}$ be semiconnected set with respect to $\eta: X \times X \times[0,1] \rightarrow R^{n} ; f: X \rightarrow R$ is said to be semipreinvex with respect to $\eta$ if and only if, for any $x, y \in X, \lambda \in[0,1], \lim _{\lambda \rightarrow 0} \lambda \eta(x, y, \lambda)=0$ and $f(y+$ $\lambda \eta(x, y, \eta)) \leq \lambda f(x)+(1-\lambda) f(y)$.

In [27], Peng and Chang presented the definition of semi$G$-preinvex functions as follows.

Definition 10. Let $X \subseteq R^{n}$ be semiconnected set with respect to $\eta: X \times X \times[0,1] \rightarrow R^{n} ; f: X \rightarrow R$ is said to be (strictly) semi-G-preinvex at $y$ with respect to $\eta$ if and only if there exist a continuous real-valued increasing function $G$ : $I_{f}(X) \rightarrow R$ and a vector-valued function $\eta: X \times X \times[0,1] \rightarrow$ $R^{n}$ such that, for all $x \in X(x \neq y), \lambda \in[0,1](\lambda \in(0,1))$, $\lim _{\lambda \rightarrow 0} \lambda \eta(x, y, \lambda)=0$ and

$$
\begin{aligned}
f(y & +\lambda \eta(x, y, \lambda)) \\
& \leq G^{-1}(\lambda G(f(x))+(1-\lambda) G(f(y)))(<) .
\end{aligned}
$$

If the inequality above is satisfied for any $y \in X$, then $f$ is (strictly) semi-G-preinvex on $X$ with respect to $\eta$. 
Remark 11. In order to define an analogous class of (strictly) semi-G-preincave functions with respect to $\eta$, the direction of the inequality in the definition of these functions should be changed to the opposite one.

Remark 12. Every $r$-semipreinvex function [23] $(r>0)$ with respect to $\eta$ is semi-G-preinvex function with respect to the same function $\eta$, where $G(t)=e^{r t}$.

Remark 13. Every G-preinvex function [26] with respect to $\eta$ is semi-G-preinvex function with respect to the same function $G$, where $\eta(x, y, \lambda)=\eta(x, y)$. However, the converse is not true.

Remark 14. Every G-convex function [24] is semi-G-preinvex function with respect to the same function $G$, where $\eta(x, y, \lambda)=x-y$. However, the converse is not true.

Remark 15. Every semipreinvex function [20] with respect to $\eta$ is semi-G-preinvex function with respect to the same $\eta$, where $G(t)=t$. However, the converse is not true.

Example 16. This example illustrates that a semi-G-preinvex function is not necessarily a semipreinvex function with respect to the same $\eta$.

Let $X=R$; it is easy to check that $X$ is a semiconnected set with respect to $\eta(x, y, \lambda)$ and $\lim _{\lambda \rightarrow 0} \lambda \eta(x, y, \lambda)=0$, where

$$
\eta(x, y, \lambda)= \begin{cases}\frac{x-y}{\sqrt{\lambda}}, & x<0, y<0, x>y, 0<\lambda \leq 1 \\ \lambda(x-y), & x \geq 0, y \geq 0, x \geq y \\ \lambda(x-y), & x<0, y<0, x \leq y \\ x-y, & x \geq 0, y \geq 0, x<y \\ -x-y, & x \geq 0, y<0, x<-y \\ -\frac{1}{2} x-y, & x<0, y \geq 0, x>-y \\ -y, & x \geq 0, y<0, x \geq-y \\ 0, & x<0, y \geq 0, x \leq-y .\end{cases}
$$

Let $f: X \rightarrow R$ be defined by $f(x)=\ln (|x|+5 / 2)$. From example 2.4 of [27], we know that $f$ is a semi-G-preinvex function with respect to $\eta$, where $G(t)=e^{t}$, but it is not a semipreinvex function with respect to the same $\eta$.

Now, we give another example that illustrates that semi$G$-preinvex functions exist.

Example 17. Let $X=(0, \pi / 2)$; it is easy to check that $X$ is a semiconnected set with respect to $\eta(x, y, \lambda)$ and $\lim _{\lambda \rightarrow 0} \lambda \eta(x, y, \lambda)=0$, where

$$
\eta(x, y, \lambda)= \begin{cases}\lambda x-y, & \text { if } x \geq y \\ x-y, & \text { if } x<y\end{cases}
$$

Let $f: X \rightarrow R$ be defined by $f(x)=\sin x$.
From the definition of semi-G-preinvexity, it is not difficult to conclude that $f$ is a semi-G-preinvex function with respect to $\eta$, where $G(t)=\arcsin t$.

In the sequel, we will use the following lemmas.

Lemma 18. Let $G: I_{f}(X) \rightarrow R$ be a continuous real-valued function; then $G^{-1}$ is increasing if and only if $G$ is increasing.

Lemma 19. Let $G: I_{f}(X) \rightarrow R$ be a continuous real-valued function. If $G$ is increasing and concave, then $G^{-1}$ is convex. If $G$ is increasing and convex, then $G^{-1}$ is concave.

\section{Properties and Characterizations of Semi-G-Preinvex Function}

In this section, we establish three interesting properties for semi-G-preinvex function, which extend and generalize the corresponding ones in the literature $[27,28]$.

Firstly, we show that the ratio of semi-G-preinvex functions is semi-G-preinvex, which extends earlier results by Craven and Mond [11] and Yang et al. [28]. Since the proof is obvious, it has been omitted in this paper.

Theorem 20. Let $X \subseteq R^{n}$ be semiconnected set with respect to $\eta$ and let $f$ and $g$ be real-valued functions defined on $X$. If $f(x) \geq 0, g(x)>0, f(x)$ is a semi- $G_{1}$-preinvex function, $-g(x)$ is a semi- $G_{2}$-preinvex function with respect to $\eta$ on $X$, where $G_{1}$ is concave and $G_{2}$ is convex. Then,

(i) $f(x) / g(x)$ is a semipreinvex function with respect to $\bar{\eta}(x, y, \lambda)=(g(y) /(\lambda g(y)+(1-\lambda) g(x))) \eta(x, y, \lambda)$;

(ii) if $\lim _{\lambda \rightarrow 0} \bar{\eta}(x, y, \lambda)=\eta^{*}(x, y)$, we also have $f(x) /$ $g(x)$ be an invex function with respect to $\eta^{*}(x, y)=$ $(g(y) / g(x)) \eta(x, y)$.

Theorem 21. Let $X \subseteq R^{n}$ be semi-connected set with respect to $\eta(x, y, \lambda) ; f: X \rightarrow R$ is a semi-G-preinvex function with respect to the same $\eta$ if and only if the set $F(f)=\{(x, u)$ : $x \in X, u \in R, f(x)<u\}$ is semiconnected with respect to $\eta^{\prime}: F(f) \times F(f) \times[0,1] \rightarrow R^{n+1}$, where

$$
\begin{aligned}
& \eta^{\prime}((y, v),(x, u), \lambda) \\
& \quad=\left\{\begin{array}{lr}
\eta(y, x, 0), & \lambda=0 ; \\
\left(\eta(y, x, \lambda), \frac{G^{-1}(\lambda G(u)+(1-\lambda) G(v))-u}{\lambda}\right), & 0<\lambda \leq 1,
\end{array}\right.
\end{aligned}
$$

for all $(x, u),(y, v) \in F(f)$.

Proof. In a similar way to the proof of Theorem 3.5 of [27], with suitable modifications, we can obtain the conclusion. 
Theorem 22. Let $X \subseteq R^{n+1}$ and $f(x)=\inf \{u: u \in R,(x, u) \in$ $X\}$, for all $x \in R^{n}$; $G$ is continuous real-valued increasing. If $X$ is a semiconnected set with respect to $\eta^{*}: X \times X \times[0,1] \rightarrow R^{n+1}$,

$$
\begin{aligned}
\eta^{*}( & (y, v),(x, u), \lambda) \\
\quad & \left\{\begin{array}{lr}
\eta(y, x, 0), & \lambda=0 ; \\
\left(\eta(y, x, \lambda), \frac{G^{-1}(\lambda G(u)+(1-\lambda) G(v))-u}{\lambda}\right), & 0<\lambda \leq 1,
\end{array}\right.
\end{aligned}
$$

for all $(x, u),(y, v) \in X$, where $\eta: R^{n} \times R^{n} \times[0,1] \rightarrow R^{n}$. Then $f: R^{n} \rightarrow R$ is a semi-G-preinvex function with respect to $\eta$.

Proof. (i) Let $0<\lambda \leq 1$, and let $x, y \in R^{n}$. Since $X$ is a semiconnected set with respect to $\eta^{*}((y, v),(x, u), \lambda)$, then, for any $(x, u),(y, v) \in X$,

$$
(x, u)+\lambda \eta^{*}((y, v),(x, u), \lambda) \in X, \quad \forall \lambda \in(0,1] .
$$

From

$$
\begin{aligned}
\eta^{*} & ((y, v),(x, u), \lambda) \\
\quad & =\left(\eta(y, x, \lambda), \frac{G^{-1}(\lambda G(u)+(1-\lambda) G(v))-u}{\lambda}\right),
\end{aligned}
$$

it follows that

$$
\begin{array}{r}
(x, u)+\lambda \eta^{*}((y, v),(x, u), \lambda) \\
=\left(x+\lambda \eta(y, x, \lambda), G^{-1}(\lambda G(u)+(1-\lambda) G(v))\right) \in X, \\
\forall \lambda \in(0,1] .
\end{array}
$$

Thus, by the definition of $f$, we have

$$
\begin{array}{r}
f(y+\lambda \eta(x, y, \lambda)) \leq G^{-1}(\lambda G(f(x))+(1-\lambda) G(f(y))), \\
\forall \lambda \in(0,1] .
\end{array}
$$

Hence, $f$ is semi-G-preinvex function with respect to $\eta$ on $R^{n}$.

(ii) When $\lambda=0$, the conclusion is correct obviously.

\section{Semi-G-Preinvexity and Optimality in Nonlinear Programming}

In this section, we consider nonlinear programming problems with inequality constraint and obtain some optimality results under semi-G-preinvexity. Meanwhile, two examples are given for illustration of the main results. Because semi$G$-preinvexity is a generalization of $G$-(pre-)invexity and (semi-)preinvexity, some of our results generalize the corresponding results of $[20,26]$.

We consider the mathematical programming problem with inequality constraint as follows:

$$
\begin{array}{ll}
\min & f(x) \\
\text { s.t. } & g_{i}(x) \leq 0, \quad i \in J=\{1, \ldots, m\}, \quad x \in X,
\end{array}
$$

where $X$ is a nonempty subset of $R^{n}, f, g_{i}: X \rightarrow R, i=$ $1,2, \ldots, m$. Denote the set of all feasible solutions for $(P)$ by

$$
D:=\left\{x \in X: g_{i}(x) \leq 0, i \in J\right\} .
$$

Theorem 23. Let the set of all feasible solutions $D$ in problem $(P)$ be semiconnected set with respect to $\eta$. If $f$ is a nonconstant semi-G-preincave function with respect to $\eta$ on $D$, then no interior point of $D$ is an optimal solution of $(P)$, or equivalently, any optimal solution $\bar{x}$ in problem $(P)$, if it exists, must be a boundary point of $D$.

Proof. If problem $(P)$ has no solution, the theorem is trivially true. Let $\bar{x}$ be an optimal solution in problem $(P)$. By assumption, $f$ is nonconstant on $D$. Then, there exists a feasible point $\tilde{x} \in D$ such that

$$
f(\tilde{x})>f(\bar{x}) .
$$

Let $z$ be an interior point of $D$. By assumption, $D$ is semiconnected set with respect to $\eta$. Then there exists $y \in D$ such that, for some $\lambda \in[0,1]$,

$$
z=\tilde{x}+\lambda \eta(y, \tilde{x}, \lambda) .
$$

Since $f$ is a semi-G-preincave function with respect to $\eta$ at any optimal solution $\bar{x}$ on $D$, then

$$
\begin{aligned}
f(z) & =f(\tilde{x}+\lambda \eta(y, \tilde{x}, \lambda)) \\
& \geq G^{-1}(\lambda G(f(y))+(1-\lambda) G(f(\tilde{x}))) \\
& >G^{-1}(\lambda G(f(\bar{x}))+(1-\lambda) G(f(\bar{x}))) \\
& =f(\bar{x}) .
\end{aligned}
$$

From the inequality above, we know that $f$ does not attain its minimum at an interior point $z$. This completes the proof of the theorem.

$$
\text { Let } U_{\delta}(\bar{x}) \text { denote a neighborhood of } \bar{x} \text { of radius } \delta \text {. }
$$

Theorem 24. Let the set of all feasible solutions $D$ in problem $(P)$ be semiconnected set with respect to $\eta$ and let $\bar{x}$ be a local minimum in problem $(P)$. Suppose that for every $\delta>0$ and for every $x \in D$ there exists $\tilde{\lambda} \in(0,1)$ such that $\bar{x}+\widetilde{\lambda} \eta(x, \bar{x}, \widetilde{\lambda}) \in$ $U_{\delta}(\bar{x})$. If $f$ is strictly semi-G-preinvex with respect to $\eta$ at $\bar{x}$ on $D$, then $\bar{x}$ is a strictly global minimum in $(P)$.

Proof. By assumption, $f$ is strictly semi-G-preinvex with respect to $\eta$ at $\bar{x}$ on $D$. Then

$$
f(\bar{x}+\lambda \eta(x, \bar{x}, \lambda))<G^{-1}(\lambda G(f(x))+(1-\lambda) G(f(\bar{x})))
$$

holds for all $x \in D(x \neq \bar{x})$ and any $\lambda \in(0,1)$. Since $\bar{x}$ is a local minimum in problem $(P)$, then there exists $U_{\delta}(\bar{x})$ such that the inequality

$$
f(\bar{x}) \leq f(x)
$$

holds for all $x \in U_{\delta}(\bar{x}) \cap D$. 
Now, let $x \in D$, such that $x \neq \bar{x}$. Since $D$ is semiconnected set with respect to $\eta$, then

$$
\bar{x}+\lambda \eta(x, \bar{x}, \lambda) \in D
$$

for all $x \in D$ and any $\lambda \in(0,1)$. By assumption, for every $\delta>0$ and for every $x \in D$, there exists $\tilde{\lambda} \in(0,1)$ such that $\bar{x}+\tilde{\lambda} \eta(x, \bar{x}, \tilde{\lambda}) \in U_{\delta}(\bar{x})$. From $(20)$, there exists $\tilde{\lambda} \in(0,1)$ such that

$$
f\left(\bar{x}+\widetilde{\lambda}_{\eta}(x, \bar{x}, \tilde{\lambda})\right) \geq f(\bar{x}) .
$$

By virtue of (19) and (22), we obtain

$$
\begin{aligned}
f(\bar{x}) \leq & f(\bar{x}+\tilde{\lambda} \eta(x, \bar{x}, \tilde{\lambda})) \\
< & G^{-1}(\tilde{\lambda} G(f(x))+(1-\tilde{\lambda}) G(f(\bar{x}))) \\
\leq & G^{-1}(\tilde{\lambda} G(\max \{f(x), f(\bar{x})\}) \\
& \quad+(1-\tilde{\lambda}) G(\max \{f(x), f(\bar{x})\})) \\
= & G^{-1}(G(\max \{f(x), f(\bar{x})\})) \\
= & \max \{f(x), f(\bar{x})\} .
\end{aligned}
$$

If $\max \{f(x), f(\bar{x})\}=f(\bar{x})$, from (23), we can get $f(\bar{x})<$ $f(\bar{x})$, which is impossible. Thus, $\max \{f(x), f(\bar{x})\}=f(x)$; it follows from (23) that the following inequality

$$
f(x)>f(\bar{x})
$$

holds. By the arbitrariness of $x$, we get the conclusion of the theorem; that is, $\bar{x}$ is a strictly global minimum in $(P)$.

Theorem 25. Let the objective function $f$ be the semi-Gpreinvex with respect to $\eta$ on $D$, and let the constraint functions $g_{i}(i \in J)$ be semi- $G_{i}$-preinvex with respect to the same function $\eta$ on $D$. Then, the set of all optimal solutions $A$ is semiconnected set with respect to the same $\eta$.

Proof. Let $x_{1}$ and $x_{2}$ be optimal solutions in $(P)$ such that $x_{1} \neq x_{2}$. Then $f\left(x_{1}\right)=f\left(x_{2}\right)=\min _{x \in D} f(x)$. By assumption, $f$ is semi- $G$-preinvex with respect to $\eta$ on $D$. Since $x_{1}, x_{2} \in D$, from Definition 10, we have

$$
\begin{aligned}
f\left(x_{2}\right. & \left.+\lambda \eta\left(x_{1}, x_{2}, \lambda\right)\right) \\
\leq & G^{-1}\left(\lambda G\left(f\left(x_{1}\right)\right)+(1-\lambda) G\left(f\left(x_{2}\right)\right)\right) .
\end{aligned}
$$

From $f\left(x_{1}\right)=f\left(x_{2}\right)$, one has

$$
\begin{aligned}
f\left(x_{2}\right. & \left.+\lambda \eta\left(x_{1}, x_{2}, \lambda\right)\right) \\
& \leq G^{-1}\left(\lambda G\left(f\left(x_{1}\right)\right)+(1-\lambda) G\left(f\left(x_{2}\right)\right)\right) \\
& =f\left(x_{1}\right) \\
& =f\left(x_{2}\right) .
\end{aligned}
$$

To prove that $x_{2}+\lambda \eta\left(x_{1}, x_{2}, \lambda\right) \in A$ for any $\lambda \in[0,1]$, it is sufficient to show that $x_{2}+\lambda \eta\left(x_{1}, x_{2}, \lambda\right) \in D$ for any $\lambda \in[0,1]$.
By assumption, $g_{i}, i \in J$, are semi- $G_{i}$-preinvex with respect to the same function $\eta$ on $D$. Therefore, for $i \in J$,

$$
\begin{aligned}
g_{i}\left(x_{2}\right. & \left.+\lambda \eta\left(x_{1}, x_{2}, \lambda\right)\right) \\
\leq & G_{i}^{-1}\left(\lambda G_{i}\left(g_{i}\left(x_{1}\right)\right)+(1-\lambda) G_{i}\left(g_{i}\left(x_{2}\right)\right)\right) .
\end{aligned}
$$

From the definition of semi-G-preinvexity, $G$ is an increasing function on its domain. Using $x_{1}, x_{2} \in D$ together with Lemma 18 , for any $\lambda \in[0,1]$,

$$
\begin{aligned}
G_{i}^{-1} & \left(\lambda G_{i}\left(g_{i}\left(x_{1}\right)\right)+(1-\lambda) G_{i}\left(g_{i}\left(x_{2}\right)\right)\right) \\
& \leq G_{i}^{-1}\left(\lambda G_{i}(0)+(1-\lambda) G_{i}(0)\right) \\
& =G_{i}^{-1}\left(G_{i}(0)\right) \\
& =0 .
\end{aligned}
$$

Thus, for any $\lambda \in[0,1]$,

$$
g_{i}\left(x_{2}+\lambda \eta\left(x_{1}, x_{2}, \lambda\right)\right) \leq 0 .
$$

So the set $A$ of all optimal solutions for $(P)$ is semiconnected set with respect to $\eta$.

Now, we illustrate the theorem above by a nonconvex optimization problem.

Example 26. We consider the following optimization problem:

$$
\begin{gathered}
f(x)=\left\{\begin{array}{ll}
\ln (3-|x|), & |x| \leq 2 \\
0, & |x|>2
\end{array} \longrightarrow \min ,\right. \\
g(x)=\arctan \left(\frac{3}{2}-|x|\right) \leq 0 .
\end{gathered}
$$

Note that the set of all feasible solutions $D=\{x \in R:-\infty<$ $x \leq-3 / 2$ or $3 / 2 \leq x<+\infty\}$ and the set of all optimal solutions $A=\{x \in R:-\infty<x \leq-2$ or $2 \leq x<+\infty\}$.

It can be proved by Definition 10 that all functions constituting the considered optimization problem are semi$G$-preinvex on $R$ with respect to the same function $\eta$, where

$$
\eta(x, y, \lambda)= \begin{cases}\lambda(x-y), & x \geq 0, y \geq 0, x \leq y, \\ \frac{x-y}{\sqrt{\lambda}}, & x \leq 0, y \leq 0, x \leq y, 0<\lambda \leq 1, \\ \lambda(x-y), & x \leq 0, y \leq 0, x>y, \\ x-y, & x \geq 0, y \geq 0, x>y, \\ -x-y, & x y<0 .\end{cases}
$$

Indeed, the objective function $f$ is semi- $G_{1}$-preinvex on $R$ with respect to $G_{1}(t)=\exp (t)-1$ and the constraint function $g$ is semi- $G_{2}$-preinvex on $R$ with respect to $G_{2}(t)=\tan t$. Since all hypotheses of Theorem 25 are fulfilled, then the set of all optimal solutions $A$ in the considered optimization problem is semiconnected with respect to the function $\eta$. 
Indeed, it is not difficult to show by Definition 8 that the set of all optimal solutions $A$ in the considered optimization problem is semiconnected with respect to the same $\eta$. However, the set of all optimal solutions $A$ is not convex.

Theorem 27. Let $y \in D$ be optimal in $(P)$. Moreover, suppose that $f$ is strictly semi-G-preinvex with respect to $\eta$ at $y$ on $D$ and the constraint functions $g_{i}, i \in J$, are semi-G $G_{i}$-preinvex with respect to the same function $\eta$ on $D$. Then, $y$ is a unique optimal solution in problem $(P)$.

Proof. By contradiction, we assume that there exists $\bar{x} \epsilon$ $D(\bar{x} \neq y)$, being another optimal solution in problem $(P)$. By assumption, the constraint functions $g_{i}, i \in J$, are semi- $G_{i}{ }^{-}$ preinvex with respect to the same function $\eta$ on $D$. It follows from Definition 10 and $\bar{x}, y \in D$, for all $i \in J$ and any $\lambda \in[0,1]$; we have

$$
\begin{aligned}
g_{i}(y & +\lambda \eta(\bar{x}, y, \lambda)) \\
& \leq G_{i}^{-1}\left(\lambda G_{i}\left(g_{i}(\bar{x})\right)+(1-\lambda) G_{i}\left(g_{i}(y)\right)\right) \\
& \leq G_{i}^{-1}\left(\lambda G_{i}(0)+(1-\lambda) G_{i}(0)\right) \\
& =0 .
\end{aligned}
$$

Then, for all $i \in J$ and any $\lambda \in[0,1]$, one has

$$
y+\lambda \eta(\bar{x}, y, \lambda) \in D
$$

Since $f$ is strictly semi- $G$-preinvex with respect to $\eta$ at $y$ on $D$, from Definition 10, the following inequality

$$
f(y+\lambda \eta(x, y, \lambda))<G^{-1}(\lambda G(f(x))+(1-\lambda) G(f(y)))
$$

holds for any $\lambda \in(0,1)$ and all $x \in D(x \neq y)$. So, we can get

$$
\begin{aligned}
f(y & +\lambda \eta(\bar{x}, y, \lambda)) \\
& <G^{-1}(\lambda G(f(\bar{x}))+(1-\lambda) G(f(y))) \\
& =G^{-1}(G(f(y)))=f(y),
\end{aligned}
$$

for $x=\bar{x}$.

Since $y+\lambda \eta(\bar{x}, y, \lambda) \in D$, we obtain that the inequality above (35) is a contradiction to the optimality of $y$ in problem $(P)$. The proof is complete.

Now, we give an example to illustrate Theorem 27 as follows.

Example 28. Consider the following optimization problem:

$$
\begin{gathered}
f(x)=\exp (\arctan |3 x|) \longrightarrow \min , \\
g(x)=\arctan (|x|-2) \leq 0 .
\end{gathered}
$$

Note that the set of all feasible solutions $D=(x \in R:-2 \leq$ $x \leq 2$ ) and the feasible solution $\bar{x}=0$ are optimal in the considered optimization problem.

From Definition 10, we can verify that the objective function $f$ is strictly semi-G-preinvex on $R$ with respect to $\eta$ and $G_{1}(t)=\tan (\ln t)$ and the constraint function $g$ is semi$G_{2}$-preinvex on $R$ with respect to $\eta$ and $G_{2}=\tan t$, where the function $\eta$ is defined by

$$
\eta(x, y, \lambda)= \begin{cases}\lambda x-\frac{y}{\sqrt{\lambda}}, & x \geq 0, y \geq 0, x<y, 0<\lambda \leq 1 ; \\ \frac{x}{2}-\frac{y}{\sqrt{\lambda}}, & x<0, y<0, x>y, 0<\lambda \leq 1 ; \\ -y, & x<0, y<0, x \leq y ; \\ \frac{x}{2}-y, & x \geq 0, y \geq 0, x \geq y ; \\ \frac{x}{3}-\frac{y}{\sqrt{\lambda}}, & x \geq 0, y<0, x<-y, 0<\lambda \leq 1 ; \\ \frac{x}{3}-\frac{y}{\sqrt{\lambda}}, & x<0, y \geq 0, x>-y, 0<\lambda \leq 1 ; \\ -y, & x \geq 0, y<0, x \geq-y ; \\ -y, & x<0, y \geq 0, x \leq-y .\end{cases}
$$

All assumptions of Theorem 27 are satisfied; by virtue of Theorem 27, the optimal solution $\bar{x}=0$ is unique in the considered optimization problem. While, in Example 26, we notice that the objective function $f$ is only semi- $G_{1}$-preinvex on $D$, but it is not strictly semi- $G_{1}$-preinvex. Therefore, the considered nonconvex optimization problem has no unique optimal solution; what is more, the set of all optimal solutions is not bounded in Example 26.

Remark 29. From Theorems 23-25, Theorem 27, and Examples 26 and 28, we can know that this class of semi-Gpreinvexity plays an important role in optimization theory and applications.

\section{Conflict of Interests}

The authors declare that there is no conflict of interests regarding the publication of this paper.

\section{Acknowledgments}

This work was supported by the Natural Science Foundation of China (nos. 11301571, 71201126, and 11271389), the Science Foundation of Ministry of Education of China (no. 12XJC910001), the Natural Science Foundation Project of Chongqing (CSTC,2012jjA00016), and the Education Committee Research Foundation of Chongqing (KJ130428).

\section{References}

[1] O. L. Mangasarin, Nonlinear Programming, Mcgraw-Hill, New York, NY, USA, 1969.

[2] M. S. Bazaraa and C. M. Shetty, Nonlinear Programming. Theory and Algorithms, Wiley, New York, NY, USA, 1979.

[3] S. Schaible and W. T. Ziemba, Generalized Concavity in Optimization and Economics, Academic Press, London, UK, 1981.

[4] D. H. Martin, “The essence of invexity," Journal of Optimization Theory and Applications, vol. 47, no. 1, pp. 65-76, 1985. 
[5] T. Weir and V. Jeyakumar, "A class of nonconvex functions and mathematical programming," Bulletin of the Australian Mathematical Society, vol. 38, no. 2, pp. 177-189, 1988.

[6] X. M. Yang and D. Li, "On properties of preinvex functions," Journal of Mathematical Analysis and Applications, vol. 256, no. 1, pp. 229-241, 2001.

[7] R. Pini, "Invexity and generalized convexity," Optimization, vol. 22, no. 4, pp. 513-525, 1991.

[8] G. M. Lee, "Nonsmooth invexity in multiobjective programming," Journal of Information \& Optimization Sciences, vol. 15, no. 1, pp. 127-136, 1994.

[9] D. Bhatia and P. Kumar, "Multiobjective control problem with generalized invexity," Journal of Mathematical Analysis and Applications, vol. 189, no. 3, pp. 676-692, 1995.

[10] S. R. Mohan and S. K. Neogy, "On invex sets and preinvex functions," Journal of Mathematical Analysis and Applications, vol. 189, no. 3, pp. 901-908, 1995.

[11] B. D. Craven and B. Mond, "Fractional programming with invexity," in Progress in Optimization, vol. 30 of Applied Optimization, pp. 79-89, Kluwer Academic, Dodrecht, The Netherlands, 1999.

[12] L. V. Reddy and R. N. Mukherjee, "Some results on mathematical programming with generalized ratio invexity," Journal of Mathematical Analysis and Applications, vol. 240, no. 2, pp. 299310, 1999.

[13] M. A. Hanson, "On sufficiency of the Kuhn-Tucker conditions," Journal of Mathematical Analysis and Applications, vol. 80, no. 2, pp. 545-550, 1981.

[14] J. Peng and X. J. Long, "A remark on preinvex functions," Bulletin of the Australian Mathematical Society, vol. 70, no. 3, pp. 397-400, 2004.

[15] B. D. Craven, "Invex functions and constrained local minima," Bulletin of the Australian Mathematical Society, vol. 24, no. 3, pp. 357-366, 1981.

[16] M. Avriel, " $r$-convex functions," Mathematical Programming, vol. 2, pp. 309-323, 1972.

[17] A. Ben-Israel and B. Mond, "What is invexity?" Australian Mathematical Society B, vol. 28, no. 1, pp. 1-9, 1986.

[18] T. Weir and B. Mond, "Preinvex functions in multiple objective optimization," Journal of Mathematical Analysis and Applications, vol. 136, no. 1, pp. 29-38, 1988.

[19] S. K. Suneja, C. Singh, and C. R. Bector, "Generalization of preinvex and B-vex functions," Journal of Optimization Theory and Applications, vol. 76, no. 3, pp. 577-587, 1993.

[20] X. Q. Yang and G. Y. Chen, "A class of nonconvex functions and pre-variational inequalities," Journal of Mathematical Analysis and Applications, vol. 169, no. 2, pp. 359-373, 1992.

[21] T. Antczak, “( $p, r)$-Invex sets and functions," Journal of Mathematical Analysis and Applications, vol. 80, no. 2, pp. 545-550, 2001.

[22] T. Antczak, " $r$-Preinvexity and $r$-invexity in mathematical programming," Computers \& Mathematics with Applications, vol. 50, no. 3-4, pp. 551-566, 2005.

[23] K. Q. Zhao, X. W. Liu, and Z. Chen, "A class of $r$-semipreinvex functions and optimality in nonlinear programming," Journal of Global Optimization, vol. 49, no. 1, pp. 37-47, 2011.

[24] M. Avriel, W. E. Diewert, S. Schaible, and I. Zang, Generalized Concavity, Plenum Press, New York, NY, USA, 1975.

[25] T. Antczak, "New optimality conditions and duality results of $G$ type in differentiable mathematical programming," Nonlinear
Analysis: Theory, Methods \& Applications, vol. 66, no. 7, pp. 1617$1632,2007$.

[26] T. Antczak, "G-pre-invex functions in mathematical programming," Journal of Computational and Applied Mathematics, vol. 217, no. 1, pp. 212-226, 2008.

[27] Z. Y. Peng and S. S. Chang, "Some properties of semi-Gpreinvex functions," Taiwanese Journal of Mathematics, vol. 17, no. 3, pp. 873-884, 2013.

[28] X. M. Yang, X. Q. Yang, and K. L. Teo, "On properties of semipreinvex functions," Bulletin of the Australian Mathematical Society, vol. 68, no. 3, pp. 449-459, 2003. 


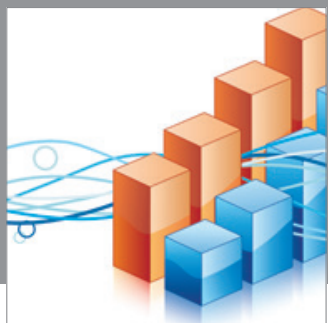

Advances in

Operations Research

mansans

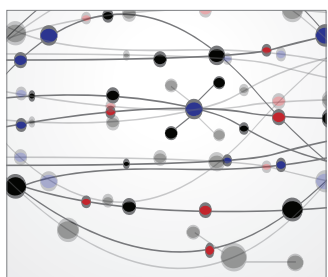

The Scientific World Journal
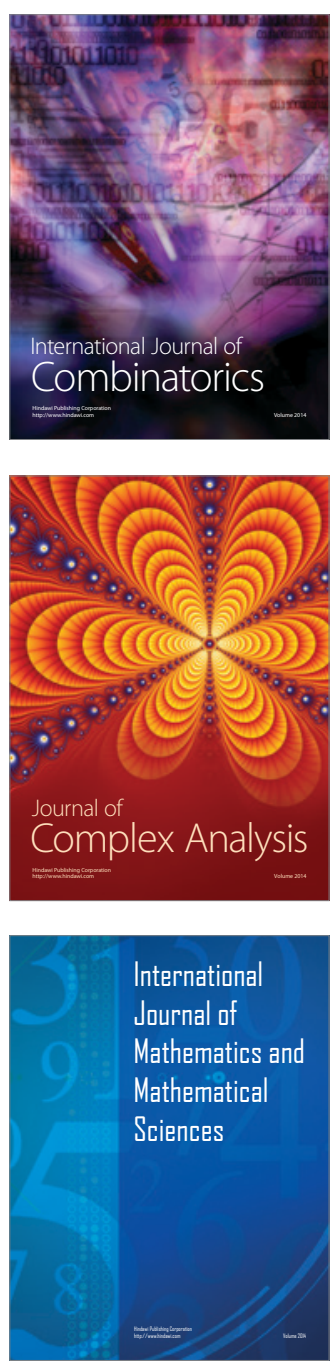
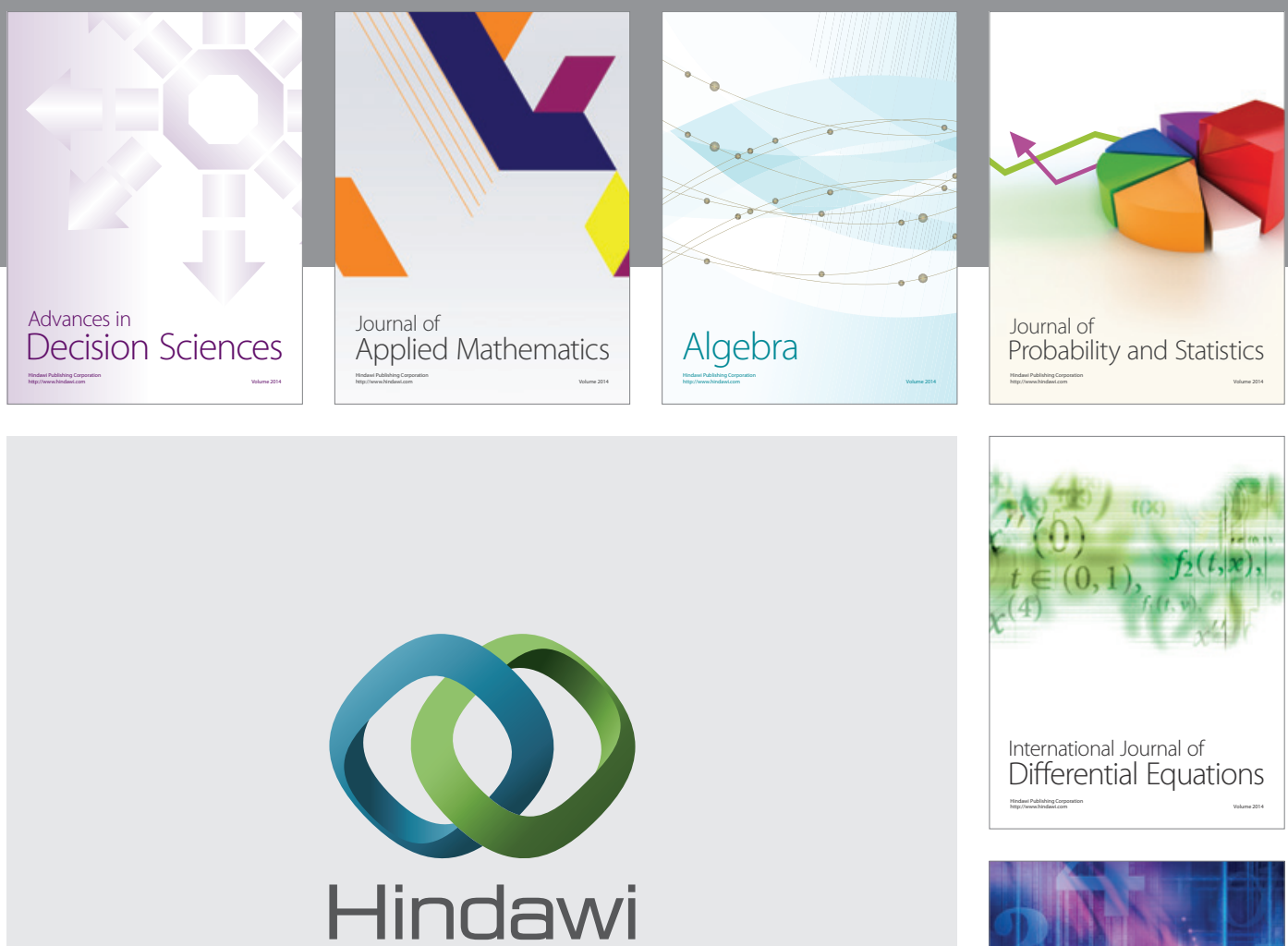

Submit your manuscripts at http://www.hindawi.com
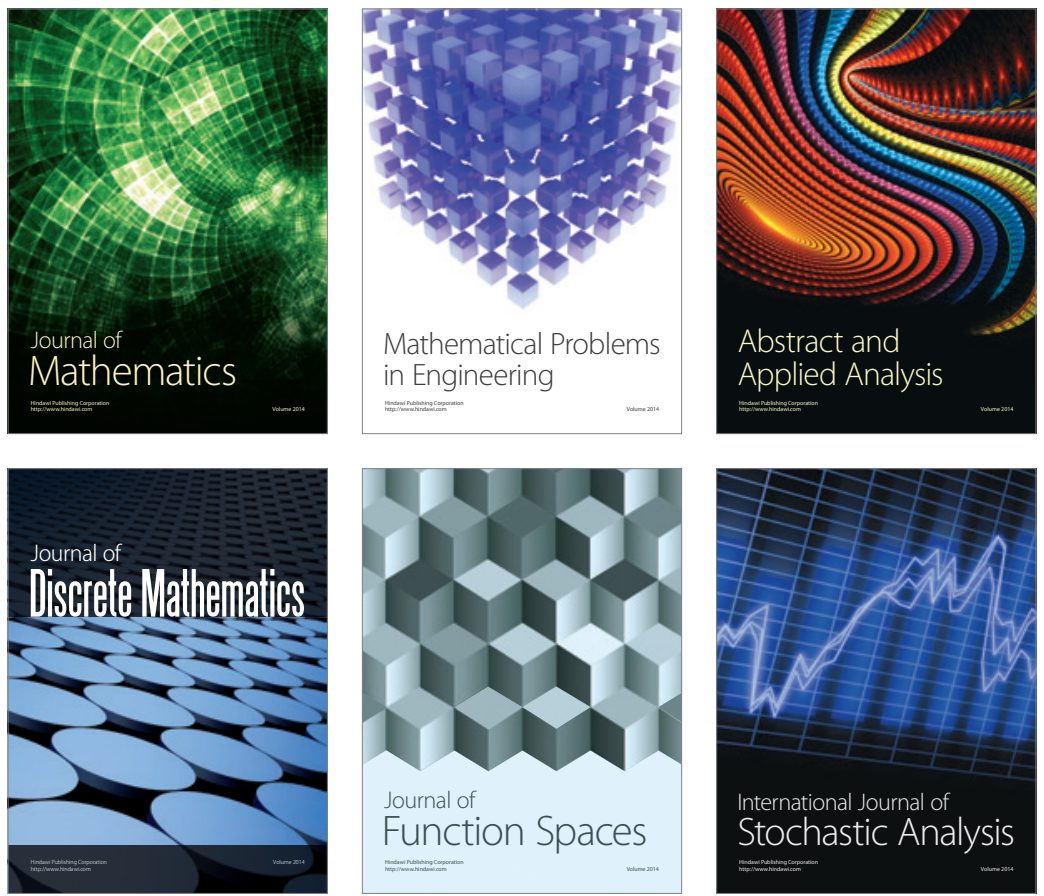

Journal of

Function Spaces

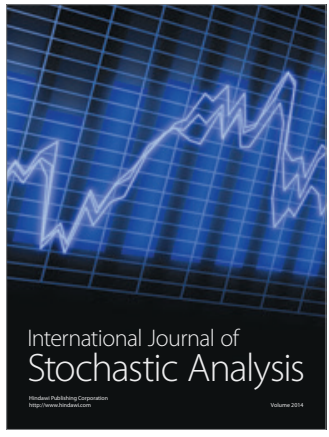

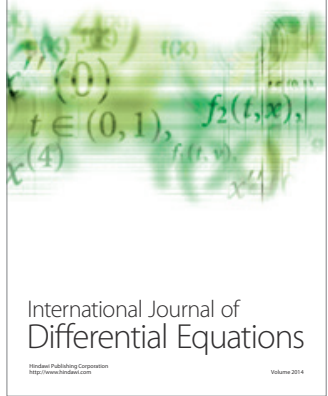
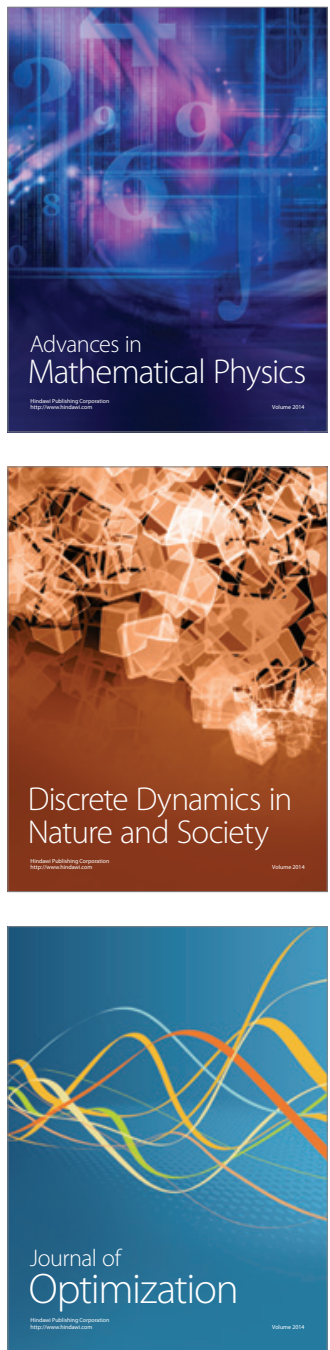